\title{
Synthesis of Diarylethynyl Methanes via a Multicomponent Coupling
}

\section{Key words}

diarylethynyl arenes

multicomponent coupling

titanocene

\section{SYNFACTrith}

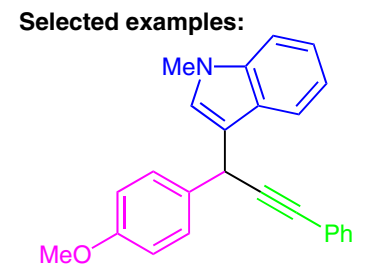

$90 \%$ yield<smiles></smiles>

$68 \%$ yield

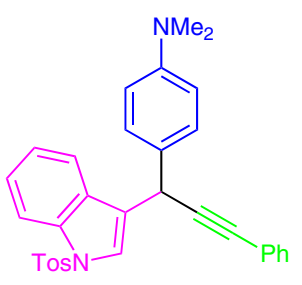

$68 \%$ yield

up to $98 \%$ yield

Significance: A straightforward synthesis of diarylethynyl methanes via a titanocene-catalyzed multicomponent coupling has been disclosed. This rapid and divergent reaction furnishes the corresponding products in good to almost quantitative yield.
Comment: The reported reaction shows a high degree of modularity and convergency in the rapid assembly of complex target molecules. Furthermore, the authors could apply the new protocol to the synthesis of a natural product.

sYNFACTS Contributors: Paul Knochel, Christoph Sämann 